\title{
Efficient and equilibrium allocations with stochastic differential utility*
}

\section{Darrell Duffie}

Stanford University, Stanford CA, USA

\section{Pierre-Yves Geoffard}

Stanford University, Stanford CA, USA

\section{Costis Skiadas}

Northwestern University, Evanston IL, USA

Submitted January 1993, accepted May 1993

This paper presents results on the existence and characterization of Pareto efficient and of equilibrium allocations in a continuous-time setting under uncertainty in which agents have stochastic differential utility, a version of recursive utility. In order to characterize equilibrium and efficient allocations in terms of pointwise first-order conditions, uniform properness conditions on preferences are avoided.

Key words: Pareto efficient allocation; Efficient allocation; Continuous time; Stochastic differential utility

JEL classification: $\mathrm{C} 62 ; \mathrm{D} 61$

\section{Introduction}

This paper presents results on the existence and characterization of Pareto efficient and of equilibrium allocations in a continuous time setting under uncertainty in which agents have stochastic differential utility, a version of recursive utility.

Section 2 is devoted to efficient allocations, including existence, characterization in terms of first-order conditions in the smooth case, and the

Correspondence to: Darrell Duffie, Graduate School of Business, Stanford University, Stanford, CA 94305, USA.

${ }^{*}$ Duffie acknowledges the support of the National Science Foundation under NSF SES 90 10062. We are grateful for discussions with Rose-Anne Dana and David Luenberger. 
associated allocation mechanism. As a by-product, we can give regularity conditions under which the state-price deflator associated with an efficient allocation is a semimartingale, a property that is useful in the characterization of security prices. Section 3 demonstrates conditions for existence of an equilibrium in which the first-order conditions for efficiency shown in section 2 apply. This approach requires us to avoid the uniform properness conditions on preferences introduced by Mas-Colell (1986).

Section 4 studies the issue of whether a local gross-substitutes condition globalizes, as it does in the additive case considered by Dana (1991). That would provide conditions for uniqueness of equilibrium and an easy existence proof.

While we deal exclusively with a pure exchange economy, the characterization of efficient allocations for production economies is of course the same, given the production choice, replacing the total private endowment with the total consumption. When specialized to exchange economies, Epstein's (1987) characterization of efficient allocations in the deterministic case is recovered here.

The remainder of this section sets up the primitives of the model.

\subsection{Consumption space}

Let $(\Omega, \mathscr{F}, P)$ be a probability space and $\mathbb{F}=\left\{\mathscr{F}_{t}: t \in[0, T]\right\}$ be a filtration of sub- $\sigma$-algebras of $\mathscr{F}$ satisfying the usual conditions. [See Protter (1990) for details.] For some given $p \in[1, \infty)$, let $D$ denote the space of consisting of all processes of the form $c: \Omega \times[0, T] \rightarrow \mathbb{R}^{l}$, with $c$ measurable with respect to the $\sigma$-algebra on $\Omega \times[0, T]$ generated by left-continuous $\mathbb{F}$-adapted processes, and with

$$
\|c\| \equiv\left[\mathrm{E}\left(\int_{0}^{T}\left\|c_{t}\right\|^{p} \mathrm{~d} t\right)\right]^{1 / p}<\infty .
$$

As usual, we identify two elements $a$ and $b$ of $D$ if $a=b$ almost everywhere. The usual positive cone of $D$ is denoted $D_{+}$. For simplicity, we assume that $\mathscr{F}_{0}$ contains only events of probability zero or one.

\subsection{The agents}

There are $m$ agents. Agent $i$ is characterized by a utility function $U_{i}$, defined on $D_{+}$by $U_{i}(c)=V_{0}^{i}$, where $V^{i}$ solves the recursive utility equation [see Duffie and Epstein (1992)]:

$$
V_{t}^{i}=\mathrm{E}\left(\int_{t}^{T} f_{i}\left(c_{s}, V_{s}^{i}\right) \mathrm{d} s \mid \mathscr{F}_{t}\right), \quad t \in[0, T]
$$


where $f_{i}: \mathbb{R}_{+}^{l} \times \mathbb{R} \rightarrow \mathbb{R}$ is a continuous function satisfying a growth condition in the first (consumption) argument and a uniform Lipschitz condition in the second (utility) argument. These technical assumptions, which can be weakened, ensure the existence of a unique $p$-integrable semimartingale ${ }^{1} V^{i}$ solving (1) for each $c$ in $D_{+}$. [See Duffie and Epstein (1992a) for $p>1$ and Antonelli (1991) for $p=1$. In a Markovian setting, without the Lipschitz condition, see Duffie and Lions (1992).] We will always assume that $f^{i}(\cdot, v)$ is strictly increasing for all $i$ and $v$, which implies that $U_{i}$ is strictly increasing [Duffie and Epstein (1992a)].

\section{Efficient allocations}

The total endowment, an element $e$ of $D_{+}$, is to be shared among the agents. The feasible allocation set is $A=\left\{\left(c^{1}, \ldots, c^{m}\right) \in D_{+}^{m}: e-\sum_{i} c^{i} \geqq 0\right\}$. For $\alpha$ in $\mathbb{R}_{+}^{m}$, let $U_{\alpha}: D_{+}^{m} \rightarrow \mathbb{R}$ be defined by

$$
U_{\alpha}(c)=\sum_{i=1}^{m} \alpha_{i} U_{i}\left(c^{i}\right)
$$

An allocation in $D_{+}^{m}$ is $\alpha$-efficient if it maximizes $U_{\alpha}$ over $\Lambda$. As is well known, if $U_{i}$ is concave for all $i$, then this definition of efficiency coincides with the usual definition of Pareto optimality: a feasible allocation $c$ is Pareto optimal if there is no other feasible allocation $\hat{c}$ such that $U_{i}\left(\hat{c}^{i}\right) \geqq$ $U_{i}\left(c^{i}\right)$, with strict inequality for at least some $i$.

Proposition 1. Suppose $f^{i}$ is concave for all $i$. Then $c$ is Pareto optimal if and only if there is some non-zero $\alpha$ such that $c$ is $\alpha$-efficient.

Proof. Duffie and Epstein (1992a) show that $U_{i}$ is a concave if $f^{i}$ is concave. The proof is then the usual application of separating hyperplanes in $\mathbb{R}^{m}$.

\subsection{Existence of efficient allocations}

Theorem 1. If $f^{t}$ is concave for all $i$ and $p>1$, then there exists an efficient allocation.

Proof. The criterion $U_{\alpha}: \Lambda \rightarrow \mathbb{R}$ is weak-upper-semicontinuous, with respect to the usual norm on $D^{m}$, since $U^{i}$ is norm-continuous and concave for all $i$. [For these properties, see Duffie and Epstein (1992).] Under the same topology, the allocation set $\Lambda$ is weak-compact, by Alaoglu's Theorem, since the lattice operations on $D$ are continuous and $\Lambda$ is convex and norm-

${ }^{1} \mathrm{~A}$ process $X$ is $p$-integrable if $\sup _{t}\left|X_{t}\right|$ is finite in $L^{p}$-norm. 
bounded. An upper semi-continuous function on a compact set achieves its supremum.

\subsection{Gradient calculation}

We will give a characterization of efficiency under differentiability. The gradient of $U_{i}$, when it exists at some $c$ in $D_{+}$, is the linear functional $\nabla U_{i}(c)$ defined by

$$
\nabla U_{i}(c) h=\lim _{\lambda \downarrow 0} \frac{U_{i}(c+\lambda h)-U_{i}(c)}{\lambda}, \quad h \in F(c) \equiv\left\{h \in D: c+h \in D_{+}\right\} .
$$

For convenience, from this point forward, unless otherwise mentioned, we take $f^{i}$ to be $C^{1}$ on the interior of its domain and take $p=2$. We let $f_{c}^{i}$ denote the partial derivative of $f^{i}$ with respect to its first (consumption) argument, and $f_{v}^{i}$ denote the partial with respect to its second (utility) argument.

We let $D_{++}$denote the quasi-interior of $D_{+}$; that is, $c$ is in $D_{++}$if $c(\omega, t) \in \mathbb{R}_{++}^{l} \equiv \operatorname{int}\left(\mathbb{R}_{+}^{l}\right)(\omega, t)$-almost everywhere.

Proposition 2. Fix $c \in D_{++}$. If $f_{c}^{i}$ satisfies a uniform growth condition in its first (consumption) argument, or if $f^{i}$ is concave and $c$ is bounded away from zero, then $\nabla U_{i}(c)$ exists and

$$
\nabla U^{i}(c) h=\mathrm{E}\left(\int_{0}^{T} \pi^{i}(c)_{t} h_{t} \mathrm{~d} t\right), \quad h \in F(c)
$$

where

$$
\pi^{i}(c)_{t}=\exp \left(\int_{0}^{t} f_{v}^{i}\left(c_{s}, V_{s}^{i}\right) \mathrm{d} s\right) f_{c}^{i}\left(c_{t}, V_{t}^{i}\right)
$$

Proof. For the case of a uniform growth condition, see Duffie and Skiadas (1991, Theorem 3). For the case of $f^{i}$ concave and $c$ bounded away from 0 , if $h \in F(c)$, then $c+\delta h \geqq \varepsilon>0$ for all $\delta$ sufficiently small, and the arguments of the proof of Theorem 2 of Duffie and Skiadas (1994) go through using concavity to bound $f_{c}^{i}\left(c_{s}+\zeta_{s}^{\delta}, V_{s}^{i}\right)$ uniformly, for $\zeta^{\delta}$ between $c$ and $c+\delta h$ for small $\delta$.

\subsection{First-order characterization of efficiency}

In principle, the first-order conditions for Pareto optimality are that 
marginal rates of substitution of the different agents coincide. We will see what that means in this setting. For the remainder of this section, we fix a feasible allocation $c \in D_{++}^{m}$ such that, for all $i, \nabla U_{i}\left(c^{i}\right)$ exists and has the Riesz representation $\pi^{i}\left(c^{i}\right)$ of (3). We let

$$
H(c)=\left\{h \in D^{m}: \sum_{i} h^{i}=0, h^{i} \in F\left(c^{i}\right), 1 \leqq i \leqq m\right\}
$$

denote the set of feasible transfers.

Proposition 3. If $c$ is $\alpha$-efficient then

$$
\sum_{i} \alpha_{i} \nabla U_{i}\left(c^{i}\right) h^{i} \leqq 0, \quad h \in H(c) .
$$

If $U_{i}$ is concave for all $i$, then the converse is true.

Proof. These are merely the first-order conditions for optimality.

Corollary. If for all $i, U_{i}$ is concave and

$$
\alpha_{i} \pi^{i}\left(c^{i}\right)=\alpha_{j} \pi^{j}\left(c^{j}\right) \text { a.e., } \quad i, j \in\{1, \ldots, m\},
$$

then $c$ is $\alpha$-efficient. Suppose that $c^{i}$ is bounded away from zero for all $i$. If $c$ is $\alpha$-efficient, than (5) holds.

Proof. For the first assertion, (5) implies (4). For the second, with $c$ bounded away from zero, $F\left(c^{i}\right)$ contains the sub-space of bounded processes. It follows that if $\pi=\alpha_{i} \pi^{i}\left(c^{i}\right)-\alpha_{j} \pi^{j}\left(c^{j}\right)$ is non-zero, there is some non-zero element $h$ of $F(c)$ such that $\mathrm{E}\left(\int_{0}^{T} h_{t} \pi_{t} \mathrm{~d} t\right)>0$. Thus (4) applies if and only if (5) does.

We give a set of sufficient conditions for an efficient allocation to be bounded away from zero that can be used as a basis for applying the last Corollary, and will also be useful later in demonstrating the existence of equilibrium. The conditions include the following 'Inada' style conditions on utilities. A special case satisfying the restriction is given by $f(x, v)=x^{\alpha}-\rho v$, for some $\alpha \in(0,1)$ and $\rho>0$.

Inada Conditions. For all $i$ :

(i) For all $x \in \mathbb{R}_{++}^{l}, \sup _{v}\left|f_{c}^{i}(x, v)\right|<\infty$.

(ii) For any sequence $x_{n}$ in $\mathbb{R}_{++}^{l}$ converging to the boundary of $\mathbb{R}_{+}^{l}$, $\inf _{v}\left|f_{c}^{i}\left(x_{n}, v\right)\right| \rightarrow \infty$. 
(iii) $f^{i}$ is concave.

Lemma 1. Suppose the Inada Conditions apply, $e$ is bounded away from zero, and $c$ is an $\alpha$-efficient allocation. Then $c$ is bounded away from zero.

The result is implied by Lemma 4, to follow in section 3. (There might seem to be a missing assumption that $\alpha$ is strictly positive, but there is a standing assumption that $c \in D_{++}^{m}$, which can only be true for $\alpha$-efficient $c$ if $\alpha \gg 0$.)

\subsection{Local versus global efficiency}

Given the consumption process $c^{i}$, its utility process $V^{i}$, and an initial weight $\alpha_{i}$, we define the discounted weight process $\lambda^{i}$ by:

$$
\lambda_{t}^{i}=\exp \left(\int_{0}^{i} f_{v}^{i}\left(c_{s}^{i}, V_{s}^{i}\right) \mathrm{d} s\right) \alpha_{i}
$$

The first-order condition (5) for efficiency can be rewritten as

$$
\lambda_{t}^{i} f_{c}^{i}\left(c_{t}^{i}, V_{t}^{i}\right)=\lambda_{t}^{j} f_{c}^{j}\left(c_{t}^{j}, V_{t}^{j}\right) \quad(\omega, t) \text {-a.e. }
$$

We therefore have regularity conditions under which an $\alpha$-efficient allocation solves, for almost every $(\omega, t)$, the problem

$$
\max _{x \in \Lambda(\omega, t)} \sum_{i=1}^{m} \lambda^{i}(\omega, t) f^{i}\left(x_{i}, V^{i}(\omega, t)\right),
$$

where $\Lambda(\omega, t)=\left\{x \in\left(\mathbb{R}_{+}^{l}\right)^{m}: x_{1}+\cdots+x_{m} \leqq e(\omega, t)\right\}$, and where $\lambda$ and $V$ are given by (6) and (7).

Proposition 3. Suppose $f^{i}$ is concave for all i. If $c$ solves (8) almost everywhere, then $c$ is $\alpha$-efficient. Conversely, suppose $e$ is bounded away from zero, the Inada conditions apply, and $c$ is $\alpha$-efficient. Then $c$ solves (8) almost everywhere.

Proof. It can easily be seen that (7) is the first-order necessary (and with concavity, sufficient) condition for (8). Then Lemma 1 and the Corollary of Proposition 2 imply the result.

\subsection{The dynamic system for efficient allocations}

We can characterize optimal allocations as the solution to a particular dynamic equation, extending the work of Lucas and Stokey (1984), Epstein 
(1987), Dana and LeVan (1989), and Kan (1990), as follows. The idea is to replace an apparently more difficult infinite-dimensional optimization problem with the finite-dimensional optimization problem (8), along with a dynamic equation for the state $(\lambda, V)$ of the optimization problem.

First, we say that $f^{i}$ is regular if it is $C^{3}$ on the interior of its domain and if, for all $v$ in $\mathbb{R}$, the Hessian matrix associated with $f(\cdot, v)$ is everywhere negative definite. (Concavity alone of $f$ assures negative semi-definiteness.) By the implicit function theorem, regularity of $f^{i}$ for all $i$ implies that there is a $C^{2}$ function $K: \mathbb{R}_{++}^{m} \times \mathbb{R}_{++}^{l} \times \mathbb{R}^{m} \rightarrow\left(\mathbb{R}_{++}^{\prime}\right)^{m}$ such that the solution to (8) is given by $K\left(\lambda(\omega, t), e(\omega, t),\left(V^{1}(\omega, t), \ldots, V^{m}(\omega, t)\right)\right)$. [We use here uniqueness of the solution to (8), implied by strict concavity of $f^{i}(\cdot, v)$, to ensure that $K$ is globally, and not merely locally $C^{2}$.] [See Mas-Colell (1985) for the details, which are standard in the study of smooth economies.]

Now we can characterize the $\alpha$-efficient allocation $c$ by $c_{t}=K\left(\lambda_{t}, e_{t}, V_{t}\right)$, where $(\lambda, V)$ solves the stochastic recursive integral equation in $\mathbb{R}^{m} \times \mathbb{R}^{m}$ :

$$
\begin{aligned}
& \lambda_{t}^{i}=\alpha_{i} \exp \left(\int_{0}^{t} f_{v}^{i}\left(K_{i}\left(\lambda_{s}, e_{s}, V_{s}\right), V_{s}^{i}\right) \mathrm{d} s\right) \\
& V_{t}^{i}=\mathrm{E}\left[\int_{t}^{T} f^{i}\left(K_{i}\left(\lambda_{s}, e_{s}, V_{s}\right), V_{s}^{i}\right) \mathrm{d} s \mid \mathscr{F}_{t}\right]
\end{aligned}
$$

Existence of solutions to such integral equations can be deduced as follows, directly from the existence of $\alpha$-efficient allocations.

Proposition 4. Suppose the Inada Conditions apply, $e$ is bounded away from zero, and, for all $i, f^{i}$ is regular. Then there exists a unique solution $(\lambda, V)$ to (9).

Proof. This immediate from Theorem 1 and Proposition 3, and the uniqueness of $\alpha$-efficient allocations implied by strict concavity.

As an alternative and more direct approach to existence of solutions (9), we could take the fixed point approach of Antonelli (1992), along the following lines. Consider the space $\mathscr{S}^{2}$ of $\mathbb{R}^{m} \times \mathbb{R}^{m}$-valued semimartingales that are finite in the norm

$$
\|X\|_{\mathscr{S}^{2}}=\left(\mathrm{E}\left[\sup _{t}\left\|X_{t}\right\|^{2}\right]\right)^{1 / 2}
$$

Then consider the map $\mathscr{X}: \mathscr{S}^{2} \rightarrow \mathscr{S}^{2}$ defined by $\mathscr{X}(\lambda, V)_{t}=\left(\hat{\lambda}_{t}, \hat{V}_{t}\right)$, where 


$$
\begin{aligned}
& \hat{\lambda}_{t}^{i}=\alpha_{i} \exp \left(\int_{0}^{t} f_{v}^{i}\left(K_{i}\left(\lambda_{s}, e_{s}, V_{s}\right), V_{s}^{i}\right) \mathrm{d} s\right) \\
& \hat{V}_{t}^{i}=\mathrm{E}\left[\int_{t}^{T} f^{i}\left(K_{i}\left(\lambda_{s}, e_{s}, V_{s}\right), V_{s}^{i}\right) \mathrm{d} s \mid \mathscr{F}_{t}\right]
\end{aligned}
$$

A fixed point of $\mathscr{X}$ is a solution of (9). This is an extension of the usual Picard iteration approach to the existence of stochastic differential equations. See, for example, Antonelli (1991). Curiously enough, Antonelli shows counterexamples to existence for analogous equations with simple linear coefficient functions, leaving this direct fixed point approach an open issue.

By considering the definition $U_{a}(e)=\sum_{i} \alpha_{i} V_{0}^{i}$ in terms of the dynamic equation (9), one can think of $U_{\alpha}$ as a generalized recursive utility function.

\subsection{Semimartingale state prices}

There are many cases, as examined in Duffie and Skiadas (1994), in which one can characterize security prices conveniently if the gradient representation $\pi^{i}\left(c^{i}\right)$ is a semimartingale. This issue can be resolved from the characterization (8) of efficient allocations, under the same regularity conditions on the aggregators $f^{1}, \ldots, f^{m}$ used in the previous section.

Proposition 5. Suppose the Inada Conditions apply, $e$ is a semimartingale bounded away from zero, and for all $i, f^{i}$ is regular. If $c$ is efficient then $\pi^{i}\left(c^{i}\right)$ is a semimartingale for all $i$.

Proof. First, $(\lambda, e, V)$ is a semimartingale in $\mathbb{R}^{m} \times \mathbb{R}^{l} \times \mathbb{R}^{m}$. Since $K$ is $C^{2}$, eq. (3) and Ito's Lemma (which implies that a $C^{2}$ function of a semimartingale is a semimartingale), we know that $\pi^{i}\left(c^{i}\right)$ is a semimartingale for all $i$.

If, in addition to the hypotheses of Proposition 5, $e$ is a special semimartingale with an absolutely continuous finite variation component, then $\pi^{i}\left(c^{i}\right)$ has an absolutely continuous finite variation component. This is important in giving a classical interpretation of short-term interest rates [the drift of $\pi^{i}\left(c^{i}\right)$ divided by $\pi^{i}\left(c^{i}\right)$, or in characterizing the behavior of security prices in general. See Karatzas et al. (1990) for an example of the 'singular' case, in which the finite variation part of $\pi^{i}\left(c^{i}\right)$ is not absolutely continuous.

\section{Equilibrium}

This section demonstrates the existence of security-spot market equilibria in which each agent's utility gradient exists and has a Riesz representation as characterized in section 2 . We will deal only with the usual notion of Arrow- 
Debreu complete markets equilibrium. As in Duffie (1986), there exists an implementation of such equilibria in a setting with security and spot markets only, given an appropriate space of admissible trading strategies, a spanning assumption on (nominal) cumulative dividend processes, and the same definition of an equilibrium. Only our utility and endowment assumptions differ from Duffic (1986). We do not require uniformly proper preferences à la Mas-Colell (1986) since the utility gradient representation used in section 2 calls for pointwise interior consumption, which is only easily guaranteed in equilibrium with an Inada condition ('infinite marginal utility at zero') and an aggregrate endowment that is bounded away from zero. In this regard, we show that the ability to dispense with uniform properness, shown in the additive-utility case by Araujo and Monteiro (1989) and Duffie and Zame (1989), has little to do with additivity, and more to do with the ability to demonstrate uniform properness when one is restricted to the set of allocations that are individually rational and pareto optimal.

Given an initial allocation $\left(e^{1}, \ldots, e^{m}\right) \in D_{+}^{m}$ with $e=\sum_{i} e^{i}$, an equilibrium is a feasible allocation $\left(c^{1}, \ldots, c^{m}\right)$ and a non-zero linear price functional $\Phi: D \rightarrow \mathbb{R}$ such that, for all $i, c^{i}$ solves the problem

$$
\max _{c \in D_{+}} U_{i}(c) \quad \text { subject to } \quad \Phi(c) \leqq \Phi\left(e^{i}\right) .
$$

For simplicity, we will examine the case of $l=1$ commodity. The case of general $l$ is easily handled by extending the following definitions and arguments.

An aggregator $f$ is defined to be good if it satisfies the uniform Lipschitz and growth assumptions and:

(i) For all $y, f(\cdot, y)$ is strictly increasing.

(ii) $f$ is continuously differentiable on the interior of its domain.

(iii) For all $x>0, \bar{\delta}_{f}(x) \equiv \sup _{v \in \mathrm{R}} f_{c}(x, v)<\infty$.

(iv) $\Lambda s x \downarrow \downarrow 0$, we have $\underline{\delta}_{f}(z) \equiv \inf _{v \in \mathbb{R}} f_{c}(x, v) \rightarrow+\infty$.

(v) $f$ is concave and continuous.

Condition (iv) is an Inada condition.

Theorem 2. Suppose the total endowment $e=\sum_{i=1}^{m} e^{i}$ is bounded away from zero and, for each $i, U_{i}$ is generated by a good aggregator $f^{i}$. Then there exists an Arrow-Debreu equilibrium $\left(\Phi,\left(c^{1}, \ldots, c^{m}\right)\right)$ with the properties:

(i) $\left(c^{1}, \ldots, c^{m}\right)$ is Pareto optimal.

(ii) For all $i, c^{i}$ is bounded away from zero.

(iii) $\Phi$ has a bounded Riesz Representation $\pi \in L$.

(iv) For each $i, U_{i}$ has a gradient at $c^{i}$ with a bounded Riesz Representation $\pi^{i}\left(c^{i}\right)$ given by (3). 
(v) For each $i$, there is a constant $k_{i}>0$ such that $\pi=k_{i} \pi^{i}\left(c^{i}\right)$.

Moreover, if $e$ is a semimartingale, then $\pi$ is a semimartingale.

The theorem is an extension of Theorem A4 of Duffie and Zame (1989). We will construct a proof based on the following series of Lemmas, closely following the approach of Appendix A of Duffie and Zame (1989).

Lemma 2. Suppose that $U$ is a stochastic differential utility function generated by a good aggregator $f$. There is a constant $k>0$ with the following property. Let $A$ be any measurable (predictable) subset of $\Omega \times[0, T]$ and let $a>0$ be any real number. If $y \in L_{+}, y \geqq a$ on $A, z \in L_{+}, z=0$ off $A$, and $z \leqq a / 2$, then

$$
U(y)-U(y-z) \leqq k \bar{\delta}_{f}\left(\frac{a}{2}\right) \mathrm{E}\left(\int_{0}^{T} z_{t} \mathrm{~d} t\right) .
$$

The proof is an application of the Mean Value Theorem and Gronwall's Inequality. Specifically, let $V$ be the utility process of $y$ and $\hat{V}$ the utility process for $y-z$. By the Mean Value Theorem, there are processes $\xi$ and $\zeta$ such that, for all $t \in[0, T]$,

$$
\begin{aligned}
V_{t}-\hat{V}_{t} & =\mathrm{E}\left(\int_{t}^{T}\left[f_{c}\left(y_{s}+\xi_{s}, V_{s}+\zeta_{s}\right) z_{s}+f_{v}\left(y_{s}+\xi_{s}, V_{s}+\zeta_{s}\right)\left(V_{s}-\hat{V}_{s}\right)\right] \mathrm{d} s \mid \mathscr{F}_{t}\right) \\
& \leqq \mathrm{E}\left(\int_{t}^{T}\left[\bar{\delta}_{f}\left(\frac{a}{2}\right) z_{s}+\alpha\left(V_{s}-\hat{V}_{s}\right)\right] \mathrm{d} s \mid \mathscr{F}_{t}\right),
\end{aligned}
$$

where $\alpha$ is a uniform Lipschitz constant on $f(c, \cdot)$. The inequality exploits the fact that $V \geqq \hat{V}$, which follows [Duffie and Epstein (1992)] from the monotonicity of $f$ with respect to consumption and the fact that $z \geqq 0$. The Stochastic Gronwall-Bellman Inequality [Appendix of Duffie and Epstein (1992)] implies that

$$
U(y)-U(y-z)=V_{0}-\hat{V}_{0} \leqq \mathrm{e}^{\alpha T} \bar{\delta}_{f}\left(\frac{a}{2}\right) \mathrm{E}\left(\int_{0}^{T} z_{t} \mathrm{~d} t\right),
$$

proving the result.

Lemma 3. Suppose that $U$ is a stochastic differential utility function generated by a good aggregator $f$. There is a constant $k>0$ with the following property. If $H$ is a predictable subset of $\Omega \times[0, T], h$ is a positive constant, and $y \in L_{+}$is such that $y \leqq h$ on $H$, then for any $\lambda \in[0, h]$,

$$
U\left(y+\lambda 1_{H}\right)-U(y) \geqq k \underline{\delta}_{f}(2 h) \lambda \mathrm{E}\left(\int_{0}^{T} 1_{H}(t) \mathrm{d} t\right) .
$$


The proof is analogous to that of Lemma 2, using the constant $k=\mathrm{e}^{-a T}$. As pointed out by Mas-Colell and Zame (1990), the following is essentially the only prerequisite for existence of Arrow-Debreu equilibrium beyond the usual technical conditions required in a finite-dimensional setting.

Lemma 4. Suppose $\left(c^{1}, \ldots, c^{m}\right)$ is a Pareto optimal allocation with $U_{l}\left(c^{i}\right) \geqq$ $U_{i}\left(e^{i}\right)$ for all $i$. Under the assumptions of Theorem 2, for each $i, c^{i}$ is bounded away from zero and $U_{i}$ satisfies the following forward cone condition at $c^{i}$ : There is some $v \in L_{+}, \varepsilon \in(0, \infty)$, and $\rho \in(0, \infty)$ such that $U_{i}\left(c^{i}+\lambda v-z\right)>U_{i}\left(c^{i}\right)$ whenever $c^{i}+\lambda v-z \in L_{+}, 0<\lambda<\rho$, and $\mathrm{E}\left(\int_{0}^{T}\left|z_{t}\right| \mathrm{d} t\right)<\lambda \varepsilon$.

Given Lemmas 2 and 3, the proof of Lemma 4 is obvious from the proof of Theorem A.4 of Duffie and Zame (1989), since the last two displayed equations substitute for eqs. (13) and (14) of Duffie and Zame (1989).

We can now complete the proof of the main existence theorem. Existence of the Arrow-Debreu equilibrium $\left(\left(c^{i}\right), \Phi\right)$ follows from Theorem A.1 of Duffie and Zame (1989), based on the same form of arguments used in Theorem A.4 of Duffie and Zame (1989). [Further details may be found in the survey by Mas-Colell and Zame (1990).] Pareto optimality of $\left(c^{1}, \ldots, c^{m}\right)$ is the First Welfare Theorem for infinite-dimensional economies. [See, for example, Mas-Colell and Zame (1990).] The fact that $\Phi$ has a bounded Riesz Representation follows from its $L^{1}$-continuity, as shown in Duffie and Zame (1989). Lemma 4 implies that, for all $i, c^{i}$ is bounded away from zero. The existence of the given gradient representation $\pi^{i}$ follows from Lemma 8 . The fact that the Riesz Representation $\pi$ of $\Phi$ is, for each $i$, a positive scalar multiple of $\pi^{i}$ is due to the Kuhn-Tucker Theorem applied to the problem (11) of each agent $i$, and the fact that the set of feasible directions from $c^{i}$ contains all bounded predictable processes (since $c^{i}$ is bounded away from zero). This implies that $\nabla U_{i}\left(c^{i}\right)$ and $\Phi$ coincide (up to a positive scaling) on the set of bounded predictable processes, which proves the last assertion of Theorem 2.

\section{Local versus global gross-substitutes}

We now explore the issue of whether a pointwise gross substitutes condition implies a global gross substitutes condition, and thereby a proof of uniqueness of efficient allocations, as well as a simple proof of existence. As shown by Dana (1991), this works with additive utility, but we shall see that this is not generally the case with recursive utility.

Under the regularity conditions given above for the existence of equilibrium, the so-called 'excess utility map' $\varepsilon: \mathbb{R}_{++}^{m} \rightarrow \mathbb{R}^{m}$ is defined by

$$
\varepsilon_{i}(\alpha)=\frac{1}{\alpha_{i}} \nabla U_{i}\left(c_{i}(\alpha)\right)\left(c_{i}(\alpha)-e^{i}\right),
$$


where $c_{i}(\alpha)$ denotes the $\alpha$-efficient allocation of consumption to agent $i$. A zero $\alpha^{*}$ of $\varepsilon$ corresponds to an equilibrium allocation $c\left(\alpha^{*}\right)$, for $c_{i}\left(\alpha^{*}\right)$ is then optimal and budget feasible for agent $i$ by the first-order condition, and markets clear.

We say that $\varepsilon$ is gross-substitutes if, for any $i$ and $k \neq i, \varepsilon_{i}$ is increasing in $\alpha_{i}$ and decreasing in $\alpha_{k}$. As shown by Dana (1991), this gross-substitutes property implies uniqueness of equilibrium (and gives a simple proof of existence).

Using the notation in (9),

$$
\varepsilon_{i}(\alpha)=\frac{1}{\alpha_{i}} \mathrm{E}\left[\int_{0}^{T} \lambda_{t}^{i} f_{c}^{i}\left(K_{i}\left(\lambda_{t}, e_{t}, V_{t}\right), V_{t}^{i}\right)\left[K_{i}\left(\lambda_{t}, e_{t}, V_{t}\right)-e_{t}^{i}\right] \mathrm{d} t,\right]
$$

where $\lambda_{0}=\alpha$. We write the integrand as $F_{i}\left(e_{t}, \lambda_{t}, V_{t}\right)$.

In the additive case, $f^{i}(c, v)=u(c)-\beta v$, we have $\lambda_{t}=\alpha$ for all $t$. Thus, as shown by Dana (1991), the Mitjushin-Polterovich conditions on $u$, which imply a gross substitutes property (in $\lambda_{t}$ ) of the 'local' excess utility map $F$, globalizes to the excess utility map $\varepsilon$. We will illustrate how this issue is complicated by relaxing additivity, even given the rather explicit dynamic system (9) for utility weights, and cven in the detcrministic case.

In the general case, the pointwise excess utility map $F$ depends nontrivially on the weights $\lambda_{t}$ and the utilities $V_{t}$ at time $t$. In order to study the effect of a change of $\alpha$ on $F\left(e_{t}, \lambda_{t}, V_{t}\right)$, one therefore needs to compute the sensitivity of $\left(\lambda_{t}, V_{t}\right)$ to a change in initial weights. In the deterministic case, this can be done through the classical technique of the resolvent matrix. ${ }^{2}$ Since the boundary conditions for (9) are a mixture of the initial condition $\lambda_{0}=\alpha$ and the terminal condition $V_{T}=0$, it makes sense to study the problem in terms of the terminal utility weights $\lambda_{T}$ rather that the initial weights. We can replace (9) in the deterministic case with

$$
\begin{array}{ll}
\lambda_{t}^{i}=\lambda_{t}^{i} f_{v}\left(K_{i}\left(\lambda_{t}, e_{t}, V_{t}\right), V_{t}\right), & \lambda_{T}=\alpha, \\
\dot{V}_{t}^{i}=-f^{i}\left(K_{i}\left(\lambda_{t}, e_{t}, V_{t}\right), V_{t}\right), & V_{T}=0 .
\end{array}
$$

Again, a zero of the modified excess utility map $\varepsilon$ defined by (12), replacing the dynamic system (9) with (13), corresponds to an equilibrium.

Using the chain rule and the form of the resolvent for (13), the sensitivity of $F\left(e_{t}, \lambda_{t}, V_{t}\right)$ to changes in $\lambda_{T}$ can be computed. Rather than trace out all of the effects, we point out an immediate obstacle to showing the gross-

\footnotetext{
${ }^{2}$ When the initial condition $\xi_{0}(\mu)$ of a dynamical system $\dot{x}_{t}=f\left(x_{t}\right), x_{0}=\xi_{0}(\mu)$ varies with some parameter $\mu$, the local change in position at time $t, \partial x_{t} / \partial \mu$ is given by $\exp \left(\int_{0}^{t} f^{\prime}\left(x_{s}\right) \mathrm{d} s\right) \partial \xi_{\mathrm{o}} / \partial \mu$. The matrix $f^{\prime}\left(x_{s}\right)$ is called the resolvent and is intensively used in the study of stability properties. See, for example, Arnold (1991) for details.
} 
substitutes property of $\varepsilon$. As a first step in such a program, it would be natural to impose conditions on $f^{i}$ under which an increase in some agent's terminal weight decreases all other agents' weights and utilities for all time. If this were true 'locally', in terms of the resolvent of the dynamic system $\left(\hat{\lambda}_{t}, \dot{V}_{t}\right)$, one might try to globalize it. For example, it would help if the exponential of a 'gross-substitutes matrix' (one with positive diagonal and non-positive off-diagonal elements) were also gross-substitutes. Even that is not true for more than two-dimensional systems. For example, to the nearest 0.1 ,

$$
\exp \left(\begin{array}{rrr}
1 & -2 & -2 \\
-2 & 1 & -1 \\
-2 & -1 & 1
\end{array}\right)=\left(\begin{array}{rrr}
17.2 & -10.1 & -10.1 \\
-10.1 & 9.7 & 2.4 \\
-10.1 & 2.4 & 9.7
\end{array}\right)
$$

As to what conditions on a resolvent imply the global gross-substitutes condition, the literature on differential equations seems to offer little guidance.

In short, going beyond the additive case, gross-substitutes conditions for even relatively explicit recursive utility functions do not seem at all obvious (to us).

\section{References}

Antonelli, F., 1991, Backward-forward stochastic differential equations, Annals of Applied Probability 3, 777-793.

Araujo, A. and P. Monteiro, 1989, Equilibrium without uniform conditions, Journal of Economic Theory 48, 416-427.

Arnold, V., 1992, Ordinary differential equations (Springer-Verlag, New York).

Dana, R.-A., 1991, Existence, uniqueness, and determinacy of Arrow-Debreu equilibria in finance models, Working paper (Laboratoire de Mathématiques Fondamentales, University of Paris VI (Jussieu), Paris).

R.A. Dana and C. LeVan, 1990, Structure of Pareto optima in an infinite-horizon economy where agents have recursive preferenecs, Journal of Optimization Theory and Applications 64, 269-292.

Dellacherie, C. and P. Mayer, 1982. Probabilities and potential B (North-Holland, Amsterdam).

Duffie, D., 1986, Stochastic equilibria: Existence, spanning number and the 'no expected financial gain from trade' hypothesis, Econometrica 54, 1161-1183.

Duffie, D. and L. Epstein, 1992a, Stochastic differential utility, Econometrica 60, 353-394.

Duffie, D. and L. Epstein, 1992, Asset pricing with stochastic differential utility, Review of Financial Studies 5, 411-436.

Duffie, D. and C.-F. Huang, 1985, Implementing Arrow-Debreu equilibrium by continuous trading of few long-lived securities, Econometrica 53, 1337-1356.

Duffie, D. and P.-L. Lions, 1992, PDE solutions of stochastic differential utility, Journal of Mathematical Economics 21, 577-606.

Duffie, D. and C. Skiadas, 1994, Continuous-time security pricing: A utility gradient approach, Journal of Mathematical Economics, this issue.

Duffie, D. and W. Zame, 1989, The consumption-based capital asset pricing model, Econometrica 57, 1279-1297.

Ekeland, I. and R. Temam, 1974, Analyse convexe et problèmes variationels (Dunod, Paris). 
Epstein, L., 1987, The global stability of efficient intertemporal allocations, Econometrica 55, $329-355$.

Geoffard, P.-Y., 1991, 'Utilité récursive: Existence d'un chemin optimal de consommation, Comptes-rendus de l'Académie des Sciences, t. 312, Série I, 657-660.

Harrison, M. and D. Kreps, 1979, Martingales and arbitrage in multiperiod security markets, Journal of Economic Theory 20, 381-408.

Holmes, R., 1975, Geometric functional analysis and its applications (Springer-Verlag, New York).

Kan, R., 1990, Structure of Pareto optima when agents have stochastic recursive preferences, Journal of Economic Theory, forthcoming.

Karatzas, I., J. Lehoczky and S. Shreve, 1990, Equilibrium models with singular asset prices, Mathematical Finance 1, no. 3, 11-30.

Karatzas, I., J. Lehoczky and S. Shreve, 1990, Existence and uniqueness of multi-agent equilibrium in a stochastic, dynamic consumption/investment model, Mathematics of Operations Research 15, 80-128

Koopmans, T., 1960, Stationary ordinal utility and impatience, Econometrica 28, 287-309.

Lucas, R. and N. Stokey, 1984, Optimal growth with many consumers, Journal of Economic Theory 32, 139-171.

Luenberger, D., 1969, Optimization by vector space methods (Wiley, New York).

Lucas, R. and N. Stokey, 1984, Optimal growth with many consumers, Journal of Economic Theory 32, 139-171.

Mas-Colell, A., 1985, The theory of general equilibrium: A differentiable approach (Cambridge University Press, Cambridge).

Mas-Colell, A., 1986, The price equilibrium existence problem in topological vector lattices, Econometrica 54, 1039-1054.

Mas-Colell, A. and W. Zame, 1992, Equilibrium in infinite-dimensional spaces, in: W. Hildenbrand and H. Sonnenschein, eds., Handbook of mathematical economics, Vol. 4 (North-Holland, Amsterdam).

Pardoux, E. and S.-G. Peng, 1990, Adapted solutions of a backward stochastic differential equation, Systems and Control Letters 14, 55-61.

Protter, P., 1990, Stochastic integration and differential equations (Springer-Verlag, New York).

Sundaresan, S., 1989, Intertemporally dependent preferences and the volatility of consumption and wealth, Review of Financial Studies 2, 73-89.

H. Uzawa, 1986, Time preference, the consumption function, and optimum asset holdings, in: J.N. Wolfe, ed., Value, capital, growth: Papers in honour of Sir John Hicks (Edinburgh University, Edinburgh). 